\title{
La Identidad Flamenca
}

Amber Judith Dirkx - Universidad de Granada

Recepción: 02.02.2021 | Aceptado: 06.06.2021

Correspondencia a través de ORCID: Amber Judith Dirkx

Citar: Dirkx, AJ (2021). La Identidad Flamenca. REIDOCREA, 10(21) 1-9.

Resumen: El flamenco se puede considerar una seña de identidad sociocultural de Andalucía con la que gran parte de la población siente una fuerte identificación. Sin embargo, no existen estudios orientados a explicar dicho vínculo o Identidad Social. En este estudio de caso, cualitativo, se investiga el concepto de la Identidad Flamenca para encontrar e indicar los factores intrínsecos que la construyen. El instrumento utilizado es la entrevista en profundidad a una participante representativa y conectada con el mundo flamenco. Los resultados indican que la Identidad Flamenca está relacionada con diferentes aspectos personales como determinados sentimientos, actitudes, raíces familiares y el estilo de vida del sujeto. Para concluir, se trata de una identidad fácilmente aplicable a una multitud de personas, lo cual fomenta la inclusión social y posiblemente pueda disminuir los estereotipos existentes en la sociedad.

Palabra clave: Identidad flamenca

\section{Flamenco Identity}

Abstract: Flamenco can be considered one of the most characteristic socio-cultural signs of Andalusia that a considering part of the population identifies itself with. However, studies oriented to explain this connection or Social Identity remain non-existent in the present. This qualitative case study investigated the concept of the so called Flamenco Identity in order to find and indicate the intrinsic factors that constitute it. The instrument used in this investigation was an in-depth interview with a representative participant, closely connected to the world of flamenco. The results indicate that the Flamenco Identity is related to different personal aspects such as feelings, attitudes, family history and roots and a certain lifestyle. This leads to the conclusion that it is an identity easily applicable on many different people, which can encourage group inclusion and possibly decrease existing stereotypes in society.

Keyword: Flamenco Identity

\section{Introducción}

En la globalización cultural actual el flamenco posiblemente se podría considerar el arte más característico y representativo de Andalucía. Gran parte de la población siente un vínculo tan fuerte con esta auténtica expresión cultural que incluso se podría hablar del arte flamenco como una seña de identidad sociocultural de Andalucía. Sin embargo, hasta el día de hoy aún no se ha contestado a la pregunta ¿existe y qué es exactamente la identidad flamenca? Para introducir la base del presente estudio primero se plantea una concepción teórica de la Identidad Social generalizada. Después se desarrollará un conocimiento básico del mundo de flamenco; su génesis y relación con la etnia gitana hasta llegar a la difusión internacional que existe en la actualidad. Al final se llega al objetivo principal de la investigación: la Identidad Flamenca. Con respecto a la pregunta planteada antes, no se trata de un concepto actualmente reconocido puesto que no ha sido objeto de estudio anteriormente. No obstante, el fenómeno está tan arraigado en la cultura andaluza e incluso ha llegado a muchos sitios fuera de España, que se considera de interés encontrar los factores intrínsecos de las personas que construyen una Identidad Flamenca y así conocer también lo que les une.

El concepto de la Identidad Social fue iniciado por el psicólogo social británico Henri Tajfel que transmitió la siguiente idea: 
Por muy rica y compleja que sea la imagen que los individuos tienen de sí mismos en relación con el mundo físico y social que les rodea, algunos de los aspectos de esa idea son aportados por la pertenencia a ciertos grupos o categorías sociales (...) la identidad social es el conocimiento de pertenecer a determinados grupos sociales junto a la significación emocional y el valor que tiene dicha pertenencia. (Tajfel, 1981, p.255)

Pues defiende que la identidad social es la parte del individuo derivado de la pertenencia al determinado grupo (Scheepers \& Derks, 2016), por ejemplo, incorporándose la identidad de "ser andaluz/a" o "ser bailaor/a" entre otras cosas. El vínculo con un grupo determinado ayuda a definir el autoconcepto, considerando los aspectos en común entre las diferentes personas como parte de la identidad propia, concediéndole cierto valor emocional.

La diferencia entre la identidad social y la identidad personal está en que la primera se basa en el grado de semejanza experimentada entre distintas personas, cuando la segunda destaca las diferencias y características individuales (Scandroglio, López y San José, 2008).

Posteriormente se ha distinguido entre tres niveles dentro de la identificación social; cognitivo, evaluativo y emocional. El primero se refiere al mero conocimiento del individuo de pertenecer a determinado grupo. El aspecto evaluativo se considera el valor positivo o negativo que el sujeto concede a la pertenencia grupal, también conocido como la autoestima grupal. Por última, la dimensión emocional consiste en la implicación emocional y el compromiso afectivo del individuo con el grupo (Ellemers, Kortekaas \& Ouwekerk, 1999).

A través de un proceso de comparación global, se categoriza como endogrupo o ingroup aquellas personas que muestran similitudes con el autoconcepto o "self" de la persona. Al contrario, las personas percibidas como diferentes serán categorizadas como exogrupo u out-group (Turner, Hogg, Oakes, Reicher \& Wetherell, 1987). Esto conlleva dos consecuencias particulares en la percepción del individuo. La categorización de uno mismo fomenta la tendencia a acentuar los aspectos en común de la persona con el endogrupo y a exagerar las diferencias con el exogrupo, lo cual incluye las actitudes, creencias y valores, reacciones afectivas, normas de comportamiento y la forma de comunicarse. Por otro lado, el efecto de la comparación social consiste en la atribución de una evaluación de valor más positivo al endogrupo y, por contrario, el exogrupo queda valorado de manera más negativa. (Hogg \& Abrams, 1988).

Los individuos que se identifican sienten una atracción fuerte hacia el grupo como un todo, independientemente del apego que existe en las relaciones entre los sujetos (Hogg \& Hardie, 1992). Además, experimentan un mayor compromiso y menos deseo de abandono del grupo, incluso en el caso de que se trate de un grupo minoritario (Ellemers, Spears \& Doosje, 1997). Como fruto de estos mecanismos se fomenta un comportamiento adaptado a la cultura y las características del grupo determinado (Ethier \& Deaux 1994; Ullah 1987).

A partir de este momento la información que se presenta es obtenida de lectura, documentaciones e instituciones de la Junta de Andalucía. Aunque no todos los datos han sido extraídos de investigación científica, se considera una información válida utilizada para abarcar el siguiente apartado teórico sobre la historia del flamenco.

El flamenco es considerado una fusión de diferentes influencias musicales de diversas culturas que se unieron en la creación de lo que hoy en día ha recibido la denominación de "flamenco". A pesar de que aún no existe un acuerdo definitivo sobre el origen exacto, 
fue en Andalucía donde empezó su desarrollo y posteriormente se convirtió en una marca sociocultural de esta zona.

El conocimiento de las diversas influencias musicales se les adscribe a los gitanos bravíos (es decir, nómadas) que finalmente dieron lugar al nacimiento de flamenco en la tierra andaluz. Aunque la base musical no es exclusivamente de la etnia gitana, la creación sí fue suya (Álvarez Caballero, 1988). Inició con su llegada a la península ibérica desde, presuntamente, India y el sureste de Europa. La lengua gitana se denomina "romaní", sin embargo, el idioma del pueblo gitano en Andalucía es el "caló", una combinación de vocabulario romaní con morfosintaxis castellana que aún se puede contemplar en la música flamenca (Gamella, Magdalena \& Adiego, 2011). Los centros geográficos principales del arte flamenco eran Cádiz, Jerez y Sevilla; específicamente el barrio gitano antiguo Triana. Al principio se les llamaba "flamencos" exclusivamente a los gitanos residentes en Andalucía y la conexión directa con la música no apareció hasta el siglo XIX cuando se formaron los cafés cantantes, a partir del 1847 en Sevilla. Empezó la fusión flamenca entre los gitanos y los andaluces en general, haciéndose más ambicioso y elaborado, pero siempre sin perder la esencia (Blas Vega, 1984). La cultura andaluza sintió todo el impacto del estilo gitano (Fraser, 2005, p. 209).

En el inicio solamente el rítmico palmoteo le acompañó al cantante, pero más adelante la guitarra y el baile enriquecieron y ampliaron el concepto de flamenco. Lo más expresado dentro del cante jondo, o más serio, son temas relacionados con el amor, lealtad, orgullo, celos, venganza, libertad, persecución, la pena y la muerte. Pero también en muchas ocasiones las canciones son alegres y animadas, como por ejemplo en las bulerías. El trasfondo emocional se contempla con fuerza en el cante y baile (Fraser, 2005).

En la actualidad ya no sólo es parte del mundo hispánico sino reconocido como una expresión de arte universal. Andalucía se puede considerar un buen ejemplo de las llamadas culturas emocionales o extrovertidas en las cuales la expresión abierta de emociones y sentimientos es valorada positivamente, en contraste con las culturas denominadas anti-emocionales en las que se huyen de manifestación directa de tal emocionalidad (Baran, 2011). La emoción lleva a la identidad, especialmente cuando es grupal se tiende a experimentar con más intensidad. Compartiendo cultura, interacción e intereses se construyen nuevas estructuras sociales (Mercer, 2014).

\section{Objetivos o hipótesis}

El objetivo del presente estudio es encontrar e indicar que es la Identidad Flamenca y conocer los factores intrínsecos que la construyen.

\section{Métodos}

\section{Diseño de investigación}

El diseño de investigación es un estudio de caso único. Se considera una herramienta valiosa de investigación en las ciencias sociales para abarcar temas que se consideran prácticamente nuevos (Martínez Carazo, 2006). Tiene varias ventajas, puesto que examina un fenómeno contemporáneo en su entorno natural, busca dar respuesta a cómo y por qué ocurre, permite informar de forma más profunda utilizando un conocimiento más detallado y estudia desde múltiples perspectivas (Chetty, 1996). En el caso del presente estudio se sigue un enfoque cualitativo-interpretativo para obtener información sobre los factores intrínsecos la Identidad Flamenca desde la vivencia de la participante del estudio de caso. 


\section{Participante}

Francisca Fernández Fernández, mujer de 58 años, es la actual directora del Centro Sociocultural Gitano Andaluz en Granada de la Junta de Andalucía. El centro se construyó en 1989, adscrito a la Consejería de Igualdad y Bienestar Social y desde entonces su objetivo ha sido fomentar el desarrollo del colectivo gitano en Andalucía. Organiza actividades sociales y culturales, entre las cuales festivales y eventos de flamenco, tanto para el pueblo gitano como para la sociedad en general, favoreciendo así la convivencia entre ambos (Centro Sociocultural Gitano Andaluz, Junta de Andalucía). La participante además es cofundadora de la asociación de mujeres gitanas "Romi" y es primera mujer gitana que se graduó en Derecho en España.

El sujeto es considerada flamenca por varios motivos; forma parte del colectivo gitano tradicional, ha vivido una fuerte influencia y dedicación dentro de su familia y sobre todo por su implicación y pasión hacia el flamenco. Incluye en su familia varias personalidades del mundo flamenco como por ejemplo Ramón Giménez Fernández o "Faraón Gitano", José Giménez o "El Moreno", Diego Ramón Jiménez Salazar o "Diego el Cigala" y su hijo guitarrista David Carmona.

\section{Instrumentos}

El estudio de caso se ha llevado a cabo a través de la entrevista en profundidad, de especial utilidad para estudiar fenómenos sociales y en la cual se le dejó al sujeto la total libertad de compartir información sobre el tema, sin limitarla en sus respuestas (Robles, 2011). Para preparar la entrevista con una estructuración mínima se construyó anteriormente una lista de preguntas a las cuales se debería responder al hacer la entrevista. Sin embargo, sirvió meramente como guía, puesto que en la realización podrían surgir preguntas adicionales con el fin de obtener la información necesaria o profundizar en cualquier tema.

\section{Procedimiento}

Lo primero que se llevó a cabo para iniciar la investigación fue la búsqueda bibliográfica, partiendo de un marco teórico más general, la Identidad Social, hasta lo más concreto, el sentimiento flamenco. Del concepto Identidad Flamenca no existe material previo, pues es lo que se pretende llegar a evaluar en este estudio. El Centro Sociocultural Gitano Andaluz facilitó varias bases de datos, libros y revistas especializadas de su fondo de documentación, archivo y biblioteca. En febrero se realizó la primera toma de contacto con la participante, para explicarle el estudio y concretar la fecha de la entrevista. La participante es una representante adecuada al estar estrechamente conectada al mundo de flamenco y por su amplio conocimiento y cultura en relación con la materia. En la entrevista, primero se anotaron los datos personales relevantes del participante, antes de pasar a las preguntas dirigidas a su experiencia personal de la Identidad Flamenca. La entrevista se llevó a cabo en mayo y fue grabado como audio con la duración de una hora y trece minutos en total.

\section{Resultados}

A continuación, se presenta una tabla los resultados obtenidos de la entrevista. Las respuestas de la participante están mostradas en la columna derecha. Al juntar la información aportada por la participante se pudo hacer una división en la columna izquierda destacando las diferentes categorías o variables emergentes. Posteriormente esta agrupación ayuda a interpretar el concepto de la Identidad Flamenca. 


\begin{tabular}{|c|c|}
\hline $\begin{array}{l}\text { IDENTIFICADORES } \\
\text { DEL SENTIMIENTO } \\
\text { FLAMENCO }\end{array}$ & FRASES TEXTUALES DE LA ENTREVISTA \\
\hline \multirow[t]{5}{*}{$\begin{array}{l}\text { COSTUMBRE } \\
\text { FAMILIAR }\end{array}$} & $\begin{array}{l}\text { Yo por regla general soy flamenca, casi todos los gitanos lo somos; es la música que se ha vivido dentro del entorno } \\
\text { familiar. }\end{array}$ \\
\hline & El flamenco está muy arraigado dentro de la familia y predomina en casi todas las fiestas; en bodas, bautizos y navidad. \\
\hline & $\begin{array}{l}\text { Por ejemplo, en la primera comunión, estuvimos todo el día de fiesta en la casa. Y cuando hablo de fiesta... es flamenco } \\
\text { siempre. }\end{array}$ \\
\hline & Fue compartirlo con toda la familia, siempre se lo pasaban muy bien cantando y bailando. \\
\hline & $\begin{array}{l}\text { A mí lo que más me gusta es bailar, sin embargo, mi hijo toca la guitarra. Posiblemente como herencia del hermano de mi } \\
\text { padre, por observar e identificarse. }\end{array}$ \\
\hline \multirow[t]{4}{*}{ RAIICES GITANA } & Casi todos los gitanos sepan hacer algo; cantar, bailar o tocar la guitarra... aun cuando no se dedican profesionalmente. \\
\hline & El flamenco es una aportación de los gitanos, pues es gitano-andaluz. \\
\hline & $\begin{array}{l}\text { Hay sentimiento de pena en la música cuando las letras hacen alusión a las persecuciones y vivencias de sufrimiento de } \\
\text { la comunidad gitana... Me identifico al escucharlo y aunque en ocasiones me que gusta cuando está bien interpretado, } \\
\text { también me transmite tristeza. }\end{array}$ \\
\hline & $\begin{array}{l}\text { También me siento viva, la nostalgia, el gusto por esa música, acordarme de los antepasados; es el fruto de transmisión } \\
\text { familiar del flamenco. }\end{array}$ \\
\hline \multirow[t]{8}{*}{$\begin{array}{l}\text { PERSONAS } \\
\text { FLAMENCAS }\end{array}$} & $\begin{array}{l}\text { No es algo que se nazca exclusivamente, también se puede aprender hoy en día cuando existe predisposición y gusto } \\
\text { hacia la música. }\end{array}$ \\
\hline & Antiguamente era todo de oído y vivir el ambiente, hoy en día ya se puede aprender y buscar información de otras fuentes. \\
\hline & $\begin{array}{l}\text { Hay muy buenos cantantes "payos", pero ojo, aunque se puede aprender, la interpretación y el transmitir sentimiento es un } \\
\text { requisito del buen flamenco. }\end{array}$ \\
\hline & Es un concepto muy arraigado en los gitanos, pero también en los andaluces e incluso latinos. \\
\hline & En los flamencos hay mucho mestizaje, ya puede ser cualquier persona, ni gitano ni payo; es artista por igual \\
\hline & Una niña, Eva la Hierbabuena, no nació en un ambiente flamenco, pero lleva el sentimiento de flamenco dentro en su baile \\
\hline & $\begin{array}{l}\text { No hay que ser gitana para ser flamenca, solamente que desde pequeño hay más predisposición porque es algo que se } \\
\text { ve más, sin embargo, en Andalucía también se puede buscar fácilmente otras maneras de formarse }\end{array}$ \\
\hline & $\begin{array}{l}\text { El caso de un amigo, que no tenía de flamenco nada, simplemente le gustaba la música y el ambiente, ahora entiende } \\
\text { mucho e incluso compró su guitarra; es un apasionado de flamenco con identidad flamenca también. La música le ha } \\
\text { transformado, se siente muy cómodo en nuestra casa, pues se identifica con nosotros y el entorno flamenco. Se siente } \\
\text { flamenco. }\end{array}$ \\
\hline \multirow[t]{5}{*}{$\begin{array}{l}\text { SENTIMIENTO } \\
\text { GRUPAL }\end{array}$} & $\begin{array}{l}\text { Mi marido y yo somos socios de la peña flamenca "La Platería", la más antigua de España, donde se interprete el cante } \\
\text { más serio o jondo }\end{array}$ \\
\hline & Granada también es una ciudad que es muy flamenca \\
\hline & Es parte de ceremonias sociales, el flamenco, es algo grupal \\
\hline & En los "tablaos" es donde verdaderamente el artista se desarrolla, ahí se aprende todo a compás \\
\hline & Participan todos, a cantar y bailar... \\
\hline \multirow[t]{9}{*}{$\begin{array}{l}\text { SENTIMIENTO } \\
\text { FLAMENCO }\end{array}$} & $\begin{array}{l}\text { Mi sentimiento depende del palo que esté interpretando, el sentimiento cambia; dentro de la música flamenca no hay } \\
\text { generalización }\end{array}$ \\
\hline & $\begin{array}{l}\text { Estás viviendo la alegría cuando la guitarra es más alegre, e incluso las personas muy flamencas, a veces terminan llorando } \\
\text { en una buena actuación }\end{array}$ \\
\hline & Flamenco es todo sentimiento. Esa alegría, y ganas de llorar por el sentimiento que transmitía, que voces más bonitas \\
\hline & Las bulerías son pura alegría \\
\hline & Sobre todo, el flamenco te transmite alegría, se te quitan las penas y se me olvidan los dolores, solamente estás disfrutando \\
\hline & $\begin{array}{l}\text { Los flamencos son introvertidos también, y a veces las emociones son reguladas a través de la música. Por ejemplo, la } \\
\text { tristeza se puede manifestar a través de la música en momentos difíciles }\end{array}$ \\
\hline & Es un sentimiento compartido, si el espectáculo ha sido bonito, la gente se anima entre ellos y ese sentimiento se transmite \\
\hline & $\begin{array}{l}\text { El duende es cuando te transmite la persona que está ejecutando para que tú lo sientes, algo que te llega a ti a lo más } \\
\text { profundo }\end{array}$ \\
\hline & $\begin{array}{l}\text { Sentimientos de éxtasis, la magia de la música. Se entiende bien, aunque no se comprende el idioma, porque es algo se } \\
\text { transmite }\end{array}$ \\
\hline \multirow[t]{10}{*}{$\begin{array}{l}\text { ACTITUDES Y } \\
\text { ESTILO DE VIDA }\end{array}$} & $\begin{array}{l}\text { La vida de una persona flamenca es muy diferente a la de otras personas, son un espectáculo, antiguamente relacionados } \\
\text { a la noche }\end{array}$ \\
\hline & Tiene una forma de pensar más informal y libre \\
\hline & $\begin{array}{l}\text { Pues por ejemplo los extranjeros que vean un espectáculo y en cuanto termine ellos se van. Diferente es cuando es entre } \\
\text { flamencos, se queda el círculo hasta más tarde, por gusto... }\end{array}$ \\
\hline & Soy capaz de quedarme hasta muy tarde cuando hay una actuación maravillosa \\
\hline & $\begin{array}{l}\text { Una vida sin flamenco para mí sería aburrida; forma parte de nuestra vida y es muy importante, no solamente en las fiestas } \\
\text { sino también en el propio cuerpo, el día a día }\end{array}$ \\
\hline & Antiguamente se vistieron flamenco, en la actualidad menos y es más bien una actitud que se lleva dentro \\
\hline & $\begin{array}{l}\text { Me identifico más con cierta forma de vestir más flamenca, como los pendientes, no llevar negro, el pelo largo, lunares y } \\
\text { flores... }\end{array}$ \\
\hline & Los flamencos tienen buena adaptación a diferentes circunstancias y saben disfrutar el momento \\
\hline & Son personas abiertas, no hay diferencia en clase; son todos músicos con arte \\
\hline & Actitud de respeto hacia la persona que actúa \\
\hline
\end{tabular}




\section{Discusión}

A partir de los resultados se puede concluir que la Identidad Flamenca es un fenómeno real que se construye a través de diferentes factores. La participante ha hecho alusión a varios aspectos tales como la familia, sus raíces, las personalidades flamencas en general, la base grupal, los sentimientos, actitudes particulares e incluso lo ha descrito como un estilo de vida. A continuación, se explicarán todos estos factores intrínsecos implicados según el sujeto del estudio de caso y después se vinculará con el marco teórico de la Identidad Social.

En primer lugar, relaciona el flamenco con el entorno familiar, ya que entró en contacto con el flamenco siendo muy joven. Le recuerda especialmente a las celebraciones y fiestas. Explica que son eventos compartidos entre todos y percibe el flamenco como una herencia dentro de la familia, que a través de la identificación se ha ido pasando de una generación a otra. Lo denomina una transmisión familiar. La segunda categoría que conecta a la Identidad Flamenca son sus raíces de gitana, afirmando la clara aportación de los gitanos en el flamenco. Además, se identifica, experimentando cierta tristeza, con las temáticas de canciones cuando describen parte de la historia del sufrimiento de los gitanos.

Sin embargo, luego aclara que el arte flamenco no está exclusivamente relacionado con el nacimiento o las raíces de la persona. Destaca que actualmente se puede aprender de diversas fuentes y que además cualquier persona lo puede conseguir con la predisposición y pasión hacia la música. Esta fusión flamenca lleva a la ampliación del endogrupo; creando unas nuevas estructuras sociales. Es decir, el flamenco ha captado el interés de muchas personas de diferentes procedencias y ya no es solamente parte de la etnia gitana. Todas las personas que se sienten fuertemente vinculadas pueden llegar a identificarse como personalidades "flamencas". La participante aporta algunos ejemplos de conocidos que no han crecido en entornos vinculados pero que sin embargo se han convertido en artistas o apasionados del flamenco. Explica cómo la música les ha transformado hasta llegar a identificarse y sentirse flamencos. Adicionalmente subraya la importancia del factor grupal, cuando el flamenco se vive dentro de tablaos y peñas flamencas y todo el mundo participa.

Un aspecto intrínseco clave del flamenco es el sentimiento. En muchas ocasiones el flamenco trata una temática estrechamente conectada con la emoción humana y los sentimientos universales como la alegría y la tristeza. Esto facilita la identificación propia con los acontecimientos vitales de las personas pues se expresan y transmiten esas experiencias. Los sentimientos concretos cambian en función de los palos de flamenco que se están interpretando. La participante añade que las personas muy flamencas, se emocionan tanto incluso hasta el punto de llorar en ocasiones. Describe el flamenco como puro sentimiento, en lo que además es capaz de olvidarse de todas las penas al vivirlo. Incluso en el caso de alguien que no entiende el castellano o el caló, asegura que el tono emocional del mensaje se transmite por ser expresado con mucha intensidad.

Por último, la Identidad Flamenca conlleva determinadas actitudes y un estilo de vida, que puede formar parte de cualquier persona. Se suele referir a un estilo de vida más nocturno, ya que una persona flamenca se queda hasta tarde en las actuaciones e incluso mucho tiempo después si lo está disfrutando. Como menciona la participante en la entrevista: "son todo un espectáculo". Esto contrasta por ejemplo con otras culturas o normas de comportamiento cuando una persona se va en cuanto termina la actuación. Además, describe el flamenco y su alegría como parte del propio cuerpo, no exclusivamente en fiestas sino por estar muy presente en la vida diaria. 
Las actitudes diferenciadoras se resumen en ser personas flexibles, que saben disfrutar el momento y tienen un fuerte respeto hacia el artista que está actuando. Añade a la descripción que se trata de personas abiertas, con una forma de pensar informal y libre. Por último, cuenta que antiguamente se vistieron de flamenco pero que actualmente lo importante es más bien la actitud que se lleva dentro. Sin embargo, sí existe identificación de su parte con cierta forma de vestir "más flamenca", refiriéndose a detalles más sutiles como los pendientes, utilizar colores, lunares y flores y llevar el pelo largo.

En el estudio de caso se observa los tres niveles de la identificación social con el endogrupo, ya que, el sujeto reconoce su pertenencia al grupo social determinado, en este caso los "flamencos", además de conceder un claro valor emocional positivo o autoestima grupal alto. Igualmente experimenta una fuerte implicación emocional y compromiso afectivo. Habla de "nosotros" e incluye las características en común en la identidad propia. También se nota la tendencia a acentuar las similitudes y concederles un valor positivo, por ejemplo, cuando menciona la inexistencia de diferenciación en clase, afirmando que todos son considerados por igual como personas con arte. Por lo contrario, se puede reconocer la existencia de un exogrupo cuando destaca que la vida de una flamenca es muy diferente a los demás, o al imaginarse una vida sin flamenco describe el aburrimiento que para ella supondría. Siente una fuerte atracción incluso cuando no hay apego directo entre las personas; explica que el sentimiento compartido durante el espectáculo provoca que las personas se animan entre ellos, disfrutando y relacionándose todos. Afirma que la sensación grupal y el hecho de compartir aumentan todavía más la intensidad de las emociones experimentadas, especialmente en el caso de la alegría. Con respecto a la cultura emocional en Andalucía, pone a Granada la etiqueta de ser una ciudad muy flamenca. Posiblemente la perspectiva de aprobación hacia la expresión de emotividad tenga influencia en el éxito y aceptación del arte flamenco esta zona.

En los estudios cualitativos más que extender y generalizar los resultados, se crea una teoría que se puede transferir a otros casos (Bickman \& Rog, 1998). Con respecto al presente estudio es importante tener en cuenta que solamente está basado en un caso particular. A pesar de ser un sujeto representativo, conviene la futura investigación de más casos, en la cual este estudio puede servir de base. La intención es extender la validez y cantidad de la información obtenida entrevistando a más personas y de diferentes culturas. Así se podrá llegar a conocer las diferencias y sobre todo similitudes en la experiencia personal de la Identidad Flamenca. La recomendación seria entrevistar a personas gitanas, y no-gitanas, andaluz y no-andaluz, para conocer los factores en común. La percepción de una identidad similar puede fomentar la inclusión cultural, creando nuevos vínculos y disminuyendo los estereotipos actuales que existen en la sociedad. Es decir, la Identidad Flamenca es en parte una actitud y un sentimiento que forma parte de muchas personas, pues puede servir como una herramienta de unión y dejar por un lado la exclusión.

\section{Referencias}

Álvarez Cabellero, A (1988). Gitanos, payos y flamencos, en los orígenes del Flamenco. Madrid: Cinterco.

Blas Vega, J (1984). Los Cafés cantantes de Sevilla. Madrid: Cinterco.

Chetty, S (1996) The case study method for research in small- and medium - sized firms. International Small Business Journal, 5.
Ellemers, N, Spears, R, \& Doosje, B (1997). Sticking Together or Falling Apart: In-Group Identification as a Psychological Determinant of Group Commitment versus Individual Mobility. Journal of Personality and Social Psychology, 72, 617-626.

Ellemers, N, Kortekaas, P, \& Ouwerkerk, JW (1999). Selfcategorization, commitment to the group and self-esteem as related but distinct aspects of social identity. European Journal of Social Psychology, 29, 371-389. 
Ethier, KA, \& Deaux, K (1994) Negotiating Social Identity When Contexts Change: Maintaining Identification and Responding to Threat. Journal of Personality and Social Psychology, 67, 234-251.

Fraser, A (2005) Los Gitanos. Barcelona: Editorial Ariel.

Gamella, JF, Magdalena Nieto, CF y Adiego, I (2011). La agonía de una lengua. Lo que queda del caló en el habla de los gitanos. Parte I. Métodos, fuentes y resultados generales. Gazeta de Antropología, 27(2), 39

Hogg, MA, \& Abrams, D (1988). Social Identifications: A Social Psychology of Intergroup Relations and Group Processes. London: Routledge.

Hogg, MA, \& Hardie, AE (1992). Prototypicality, Conformity and Depersonalized Attraction: A Self-Categorization Analysis of Group Cohesiveness. British Journal of Social Psychology, 31, 41-56.

Martinez Carazo, PC (2006), El método de estudio de caso: Estrategia metodológica de la investigación científica. Pensamiento y Gestión, 20, 165-193.

Bickman, L, \& Rog, DJ (1998), Handbook of Applied Social Research Method. Thousand Oaks: Sage.

Mercer, J (2014). Feeling Like a State: Social Emotion and Identity. Cambridge University Press, 6(3), 515-535.
Scandroglio, B, López Martínez, JS y San José, MC (2008). La Teoría de la Identidad Social: una síntesis crítica de sus fundamentos, evidencias y controversias. Psicothema, 20(1), 8090.

Scheepers, D, \& Derks, B (2016). Revisiting social identity theory from a neuroscience perspective. Current opinion in Psychology, $11,74-78$.

Stets, JE, \& Burke, PJ (2000). Identity Theory and Social Identity Theory. Social Psychology Quarterly, 63(3), 224-237.

Tajfel, H (1981). Human groups and social categories. Cambridge: Cambridge University Press.

Turner, JC, Hogg, A, Oakes, PJ, Reicher, SD, \& Wetherell, MS (1987). Rediscovering the Social Group: A Self-Categorization Theory. New York: Basil Blackwell.

Robles, B (2011). La entrevista en profundidad: una técnica útil dentro del campo antropofísico. Cuicuilco, 18(52).

Ullah, P (1987). Self-Definition and Psychological Group Formation in an Ethnic Minority. British Journal of Social Psychology, 26, 1723. 


\section{ANEXO 1: PREGUNTAS DE LA ENTREVISTA}

- ¿Por qué se considera flamenca?

- ¿Cómo surgió su pasión por el flamenco, actúas, participas o contemplas?

- ¿Cómo ha vivido el flamenco desde la infancia?

- ¿Y dentro del ambiente familiar?

- ¿Qué significa el flamenco para usted?

- ¿Consideras el flamenco como un "todo" o hay algún aspecto más importante que otro (toque, cante, baile...)?

- ¿Qué es el duende y el quejido?

- ¿Es una sensación compartida o más bien individual/personal?

- ¿Cómo es la vida de una flamenca?

- ¿Es algo que se lleva en la sangre, desde pequeño, o que también se puede aprender?

- ¿Pesa más la técnica o poner "el alma"; como algo espontáneo o practicado?

- ¿Qué siente al escuchar flamenco?

- ¿Qué hace que se identifica con ello?

- ¿Qué diferencias siente que pueda haber con personas no flamencas?

- ¿Entre el cante jondo (trágico) y fiestero (alegre), qué diferencia experimenta en la manera de vivirla?

- ¿Hay algún tipo o aspecto de flamenco que se vive con más intensidad, y qué sentimientos de provoca?

- ¿Cuáles son los temas más comunes y que transmiten?

- ¿Cómo sería su vida sin el flamenco, que le faltaría? 